\title{
A conceptual approach to the formation of paramagnetic nanospecies of noble metals
}

\author{
Victor Kislyuk ${ }^{*}$, Andrii Melnyk ${ }^{* *}$, \\ Yuliia Bondar $^{* * *}$, Volodymyr Trachevskij ${ }^{* * * *}$
}

\begin{abstract}
The general concepts are analyzed regarding the approach for the formation of paramagnetic species of noble metals, with a non-rigid (labile) molecule being used as a supporting matrix. The formation of the metal nanospecies follows three stages: (i) the metal ions are captured by the matrix, (ii) the reducing agent causes formation of individual atoms separated by the matrix fragments, (iii) the individual atoms agglomerate due to conformational transformations of the labile molecule-matrix. This algorithm is realized in two distinct systems: Ag-containing nanospecies embedded within the system of polyacrylic acid (PAA) chains grafted to the film of fluorinated ethylene propylene copolymer (FEP) and Au-containing nanospecies in the free matrix of tannin-citrate- oxo-hydroxo aluminate. The evolution of the electron paramagnetic resonance (EPR) spectra while cooling down demonstrates the appearance of the exchange interaction which is suppressed at higher temperatures by the vibrational modes of the molecule-matrix. The role of the oxo-hydroxo aluminate form is one of a molecular motor sorting the individual nanospecies by their size and charge state.
\end{abstract}

K e y w o r d s: EPR, nanoparticles, paramagnetism, molecular motor, SEM

\section{Introduction}

Noble metal nanospecies attract attention both researchers from various fields [1-3] (biologists, chemists, physicists) and practitioners [4-6] (medical professionals, pharmacologists, engineers). The reasons of a permanent interest to them are their bio-compatibility (in most cases); appearance of new optical and magnetic properties absent in the bulk materials, relatively simple and low cost technology of their fabrication. However, although there is a tremendous experience in this field, the issue on developing a reproducible technology remains actual for designing nanospecies with a certain set of predefined parameters such as size, content and charge state.

Among the mechanisms describing the processes of nanoparticle self-arrangement during the reduction process in solution are the following: classical nucleation model of Becher and Döring [7] followed by La Mer's theory [8] and later by DLVO (Derjaguin-Landau-VerweyOverbeek) theory $[9,10]$; dense droplet mechanism [11] and others. The mechanisms listed describes the reduction process in non-equilibrium conditions, and the selfarrangement is the result of the multifold and mutually competitive processes determined by the reduction rate, diffusivity and nature of the stabilizing reagent, $i e$ the kinetics parameters rather than thermodynamical ones.

Further improvement of the technology demanded additional techniques for the structure formation and structure fixation, which would have been not so dependent on local stochastic processes able to turn the process in a wrong direction, eg provoking aggregation.

The application of a supporting matrix is one of the revolutionary approaches to guarantee the separation of the nanospecies [12 - 14]. One of the first realizations of this approach is the application of nanoporous structures. The principle peculiarity of the fabrication processes in the case is the possibility for separation of initial ions prior to their reduction. However, there is the risk for them to remain non-reduced due to steric obstacles caused by their immobilization on the matrix surface.

A possibility to increase the reduction rate of the separated ions is to apply a labile supporting macromolecule with additional level of freedom for conformational modulations. The macromolecule grafted to a rigid frame catches the metal ions which being reduced compile into metallic nanospecies, Fig. 1(a). Evidently, the metal clusters have mosaic structure consisting of individual groups of the reduced atoms separated by the fragments of the labile matrix.

The rigid frame is not obligatory to produce more uniform structure of the nanospecies. A supporting molecule would ensure the ion fixation even in its free state in the solution, which is advantageous for the reduction process. The macromolecule segments catch atoms which unite together increasing thereby the clustering probability, Fig. 1(b). At any rate, the ions captured within the matrix can form the clusters after their reduction.

\footnotetext{
*Institute of Metal Physics, National Academy of Sciences of Ukraine, viktor.kislyuk@gmail.com, **Institute for Sorption and Endoecology Problems, National Academy of Sciences of Ukraine, *** Institute of Environmental Geochemistry of National Academy of Sciences of Ukraine, ****Technological center of National Academy of Sciences of Ukraine
} 


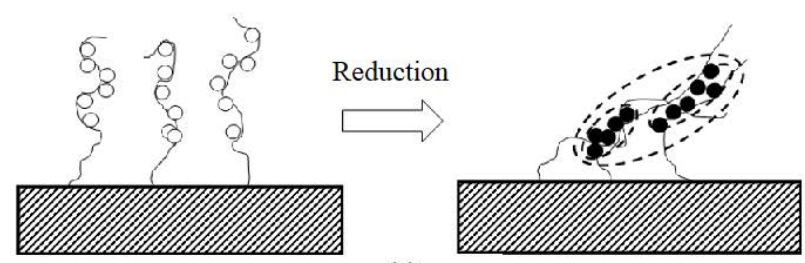

(a)

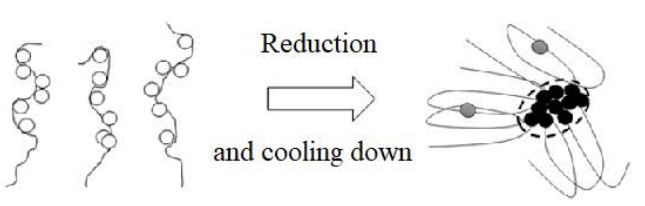

(b)

Fig. 1. Schematic illustration to the formation of metal nanoclusters with a macromolecule as a supporting matrix: (a) - macromolecule grafted to a rigid frame, (b) - macromolecule in a solution with a degree of freedom to modify its conformation depending on temperature

Thus, the increase of the reducing ability provokes the formation of the clusters in the supporting matrix. This process can be tuned toward the directed formation of the nanospecies of a certain size and charge by Brownian motor. This term, first introduced by Feynman [15], presumes the ratchet-potential [16] whose moving fragments sort the nanospecies in accordance with their size and charge. Solutions of oxy-hydrate forms of metals are found to be dynamical systems with potential profiles whose fluctuations cause local motion of charges and their self-ordering. This process is observed as short intensive fluctuations of electric current $[17,18]$. In addition, one of the possible ways to increase the reduction yield is to irradiate the reactive solution with high-energy quanta for free electron generation in the matrix $[19,20]$.

The principal objective of this research is comparative study of two distinct techniques for the formation of the metal nanospecies. They are based on the same idea of labile (not rigid) supporting molecules. The objects of the study are two systems.

\section{First (grafted matrix)}

Silver ions captured by the polyacrylic chains grafted from one end to $125 \mu \mathrm{m}$ thick rigid film of poly(tetrafloroethylene-hexafloropropylene), DuPont (FEP) with further reduction till the formation of the metal clusters.

\section{Second (unbound matrix)}

The nanoparticles formed in the colloid solution after the reduction of gold ions in the labile matrix of tannincitrate with oxo-hydrate form of aluminum as Brownian motor.

The temperature dependent conformational modifications were monitored via electron paramagnetic resonance spectra (EPR) reflecting the changes of the local interactions between adjacent nano-fragments with acquired paramagnetic properties, while the matrix materials for both systems being EPR-silent.

\section{Experiment}

\subsection{Nanospecies in the rigid matrix under irradiation}

Fluorinated polymer film as fluorinated ethylene propylene copolymer (FEP) with a thickness of $125 \mu \mathrm{m}$ was purchased from Du Pont and used as a base polymer to manufacture silver nanoparticles-loaded polymer film. Reagent grade acrylic acid monomer (Sigma-Aldrich), extra pure silver nitrate (Duksan Pure Chemicals Co., Ltd.,
Korea), sodium formate ( $>99.0 \%$, Sigma-Aldrich) were used as received. Distilled water was used throughout the experiments.

The composite material based on the FEP film with chemically bound Ag particles was synthesized through a three-stage experiment. At the first stage, the chemically inert FEP base was activated through the radiationinduced graft polymerization of acrylic acid monomer (AA) for the introduction of chemically active carboxyl groups onto the surface of FEP films through covalent bonding between grafted polyacrylic acid (PAA) chains and FEP base [21]. Grafted samples with a medium value of AA grafting degree (60\% to $120 \%$ ) were taken for the experimental work. The second stage consisted of $\mathrm{Ag}^{+}$ ions loading onto PAA chains grafted to the FEP film (FEP-g-PAA) followed by the in situ formation and stabilization of $\mathrm{Ag}$ nanoparticles within the grafted layer. For this purpose, the FEP-g-PAA film was immersed in $0.1 \mathrm{M} \mathrm{AgNO}_{3}$ solution for $12 \mathrm{~h}$. After filtration, washing with distilled water, and drying at middle temperature, the resulting FEP-g-PAA $\left(\mathrm{Ag}^{+}\right)$film was added to $0.5 \%$ solution of sodium formate for reduction of silver ions (the third stage). Finally, the silver nanoparticles-loaded FEP film was separated by filtration, washed with deionized water, and dried at $60^{\circ} \mathrm{C}$ for $24 \mathrm{~h}$.

\subsection{Nanospecies in the labile matrix with oxyhydrate molecular motor}

$\mathrm{Au} / \mathrm{Al}$ nanocomplex was synthesized by co-reduction method [22] modified according to peculiarities of the redox processes [23]. A water solution of the chlorine containing compounds of $\mathrm{Au}$ and $\mathrm{Al}\left(\mathrm{HAuCl}_{4}\right.$ and $\left.\mathrm{AlCl}_{3}\right)$ was used as a precursor ("metal containing solution"). To monitor the paramagnetic properties of a nanosystem the solid fracture was extracted from the solution and dried at temperatures below $50^{\circ} \mathrm{C}$ by various ways: i) centrifugation and collection of the solid sediment; ii) salting out from the water solution of $\mathrm{Au} / \mathrm{Al}$ composite in dioxane; iii) lyophilic drying of the solution with increased $\mathrm{Al}$ content; iv) lyophilic drying of the $\mathrm{Au} / \mathrm{Al}$ solution with lack of the reducing agent.

\section{$2.3 \mathrm{EPR}$}

EPR spectra were recorded on a X-band spectrometer Bruker Elexsys E580 at the temperature range 125 - 325 K. EPR spectra recording conditions were as follows: frequency -within $9.3-9.9 \mathrm{GHz}$; microwave power $10-20$ 
$\mathrm{mW}$ in the case of golden nanoparticles and $2-6.3 \mathrm{~mW}$ for silver ones; modulation intensity -correspondingly 10 and $0.4-1 \mathrm{G}$ with modulation frequency $100 \mathrm{kHz}$, time constant $-81 \mathrm{~ms}$, sweep time - $180 \mathrm{~s}$, number of scans - 4 (for golden nanoparticles). Before spectral investigation samples of the studied nanocomposites were placed into standard $3 \mathrm{~mm}$ quartz ampoule (Norrell). Sample of diphenylpicrylhydrazyl (DPPH) with $g=2.0036$ was used for the spectrometer calibration. Variable temperature unit BVT3000 was used for temperature measurements, every temperature value was set for at least 3 minutes, the stability of measurements was $\pm 0.5 \mathrm{~K}$.

\subsection{SEM}

The surface morphology of the silver nanoparticlesloaded FEP films was recorded by a Hitachi S-4100 field emission scanning electron microscope (SEM; Hitachi, Ltd., Tokyo, Japan) at an acceleration voltage of $15 \mathrm{keV}$. The elemental composition was performed by energydispersive X-ray spectroscopy (EDS). The studied samples were sputter-coated with a thin Pt layer prior to examination.

\section{Results and discussions}

\subsection{EPR of silver nanospecies in the grafted matrix}

EPR spectra of silver nanospecies in the grafted matrix, Fig. 2, consist of the superposition of two signals (forms $\mathrm{A}$ and $\mathrm{B}$, respectively) with correspondent values of $g$-factors: $g_{\perp} \sim 2.036, g_{\|} \sim 2.02, g_{0}^{*}=2.031$ (calculated with formula under Table 1$) ; g_{\text {eff }}=2.03, \Delta B=7$ mT.

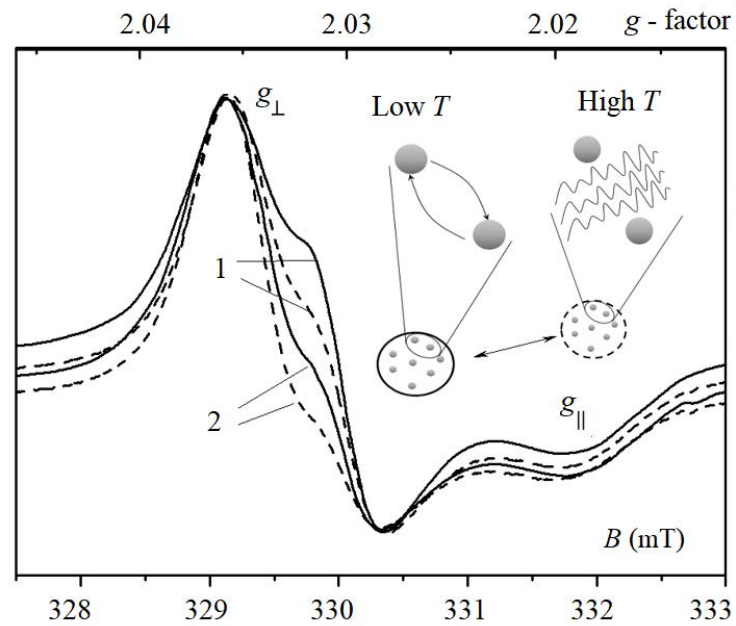

Fig. 2. EPR spectra of silver nanospecies with prolonged (1) and round (2) shapes in the polyacrilic matrix grafted to the FEP film measured under various temperatures: $300 \mathrm{~K}$ (dashed line) and 275 $\mathrm{K}$ (solid line). Inset: Schematic illustration explaining the role of the exchange interaction.

The behavior of the spectra while temperature decreases from the room temperature to $225 \mathrm{~K}$ reflects the redistribution of the contributions from paramagnetic particles: intensity of the line attributed to the paramagnetic centers with $g_{\mathrm{eff}}=2.03, \Delta B=7 \mathrm{mT}$ increases which is accompanied by the decrease of the signal with parameters of spin-Hamiltonian characteristic for axial symmetry. Evidently the anisotropic signal belongs to prolonged species and anisotropic one does to spherical (Fig. 2). The particle form (prolonged or spherical), is, likely, the consequence of the concentration-dependent structure formation with spatial organization of AA-FEP fragments being the structure forming factor, Fig. 1(a). Further increase of $\mathrm{Ag}$ concentration gives rise to joining the nanospecies into bigger agglomerates. Inset in Fig. 1 illustrates the individual nanospecies (dashed lines) directed by the structure of the polymer chains, which can be followed by the further aggregation into coarser clusters and so on.

The analysis of the SEM-images in detail demonstrates a mosaic structure of the nanospecies embedded in the matrix, as it is presumed for such a system with the matrix fixed from one end. Some fragments of this mosaic are separated by the polyacrylic chains. The similar behavior is also reported in other papers [24]. The set of sterically close metallic nanospecies manifests itself in EPR as an individual unit provided there is an exchange interaction between them which can be suppressed under higher temperatures due to vibrational modes of the separating fragments of the matrix molecule (Fig. 2, inset). Thus, the lower temperature the higher yield produced by this set into the intensity of the EPR signal with $g_{\text {eff }} \sim 2.03$ attributed to the collective response of the adjacent nanospecies. In contrary the temperature increase destroys their spin coherency, and intensity of this signal reduces. Hence the signal with $g_{\text {eff }} \sim 2.03$ is associated with the nanospecies comprised of individual finer nanoparticles separated from each other not more than distance sufficient for an effective spin exchange, while the anisotropic nature of comprising individual centers is hampered.

Individual isolated silver atoms are identified in the EPR spectra as signals with characteristic fine structure attributed to interaction of electron magnetic momentum with nucleus momentum $(J=1 / 2)$ [25], which is not observed in this study and, hence, is the evidence of $\mathrm{Ag}_{\mathrm{n}}$ clusters as only paramagnetic centers in the matrixes.

The EPR spectra transform at temperatures below 200 $\mathrm{K}$ with a new anisotropic feature, form $\mathrm{C}$ being appeared, see Fig. 3(b) and Tab. l. At the same time $200 \mathrm{~K}$ temperature is known to be the point of phase transition for the water embedded within nanopores or in the interchain medium [26]. This is the reason to attribute this transformation of the EPR signal to the phase transition of water which is present at a sufficient extent (with $80 \%$ degree of hydration) and bound to the carboxylic groups via H-bonding [27]. The water freezing is assumed to be responsible for the modification of the spatial organization of the molecules in the supporting matrix. The latter reconstructs the system of the hydrogen bonds, which 

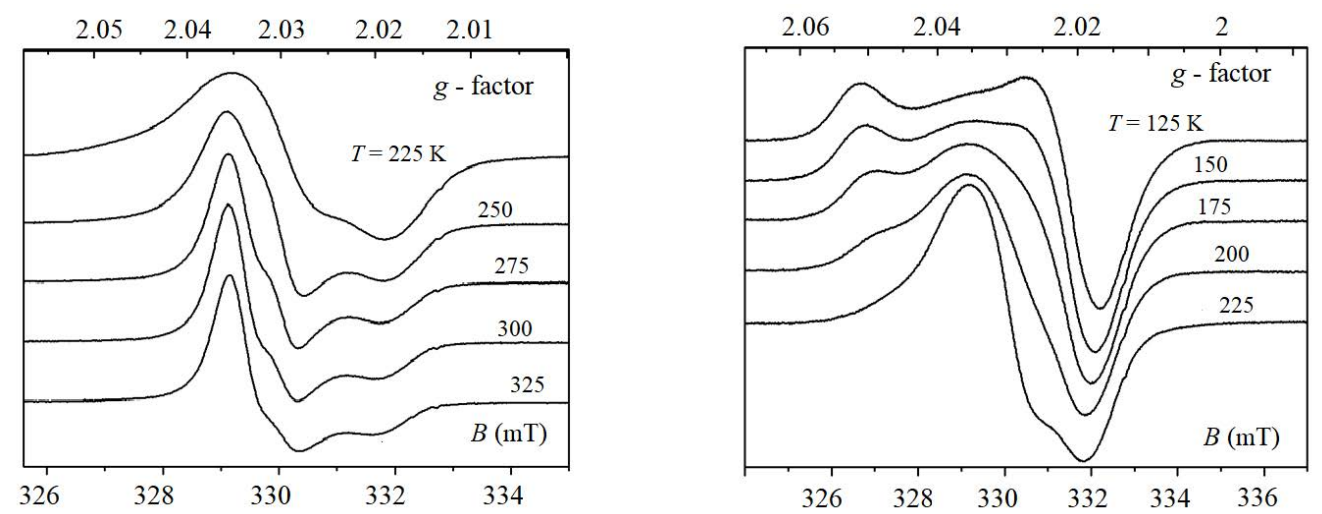

Fig. 3. Evolution of the EPR spectra of the system containing silver particles in polyacrilic matrix grafted to the FEP measured within the temperature ranges: (a) $-325-225 \mathrm{~K}$, and (b) - 225-125 K
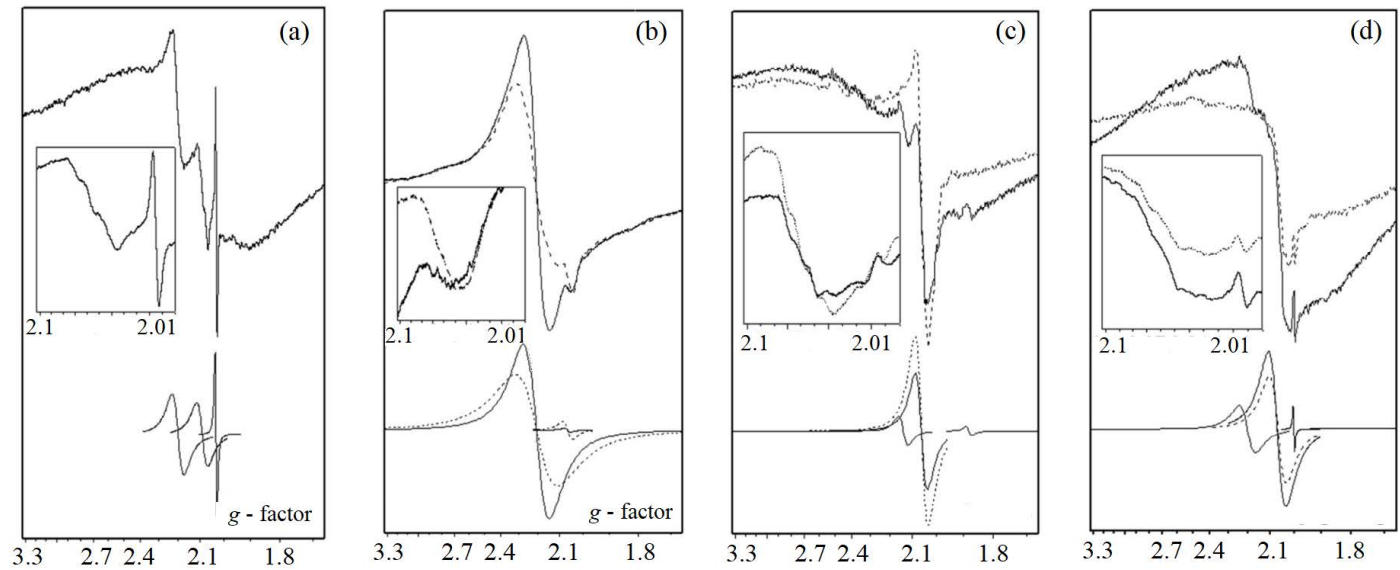

Fig. 4. EPR spectra of gold-containing samples prepared by various methods: (a) - centrifuged from the solution of stoichiometric content, (b) - salted-out in dioxane, (c) - lyophilic drying of the reactional mixture with aluminum excess, and (d) - lyophilic drying of the solution with non-stoichiometric content of the reducing agent

causes the change in the polyhedron of the coordination vicinity of silver atoms.

A quantitative analysis of the forms of the EPR lines, in particular the proximity of the average values of $g$ (Tab. 1) points to almost similar spin density localized at silver atoms, which is an evidence of a similar nature of donating environment. $g_{0}^{*}$ calculated as $g_{0}^{*}=\left(2 g_{\perp}+\right.$ $\left.g_{\|}\right) / 3$.

Table 1. EPR parameters of the Ag-containing centers (forms)

\begin{tabular}{lccccc} 
Form & $\begin{array}{c}g_{\|} \\
(-)\end{array}$ & $\begin{array}{c}g_{\perp} \\
(-)\end{array}$ & $\begin{array}{c}g_{0}^{*} \\
(-)\end{array}$ & $\begin{array}{c}g_{\text {eff }} \\
(-)\end{array}$ & $\begin{array}{c}\Delta B \\
(\mathrm{mT})\end{array}$ \\
\hline $\mathrm{A}(300-275 \mathrm{~K})$ & 2.019 & 2.036 & 2.030 & - & - \\
$\mathrm{B}(300-175 \mathrm{~K})$ & - & - & - & 2.03 & 7 \\
$\mathrm{C}(200-175 \mathrm{~K})$ & 2.052 & 2.017 & 2.029 & - & - \\
\hline
\end{tabular}

$3.2 \mathrm{EPR}$ of the gold nanospecies in unbound matrix of tannin-citrate-oxo-hydroxo aluminate

The reduction of gold ions in the system of aluminum oxohydrate-tannin-citrate causes paramagnetic proper- ties revealed in the EPR spectra as four various signals with $g$-factor values 2.17, 2.062, 2.0048 and $g<2$, which indicates the presence of, respectively, ions of twovalent gold, gold-containing clusters with odd number of atoms, radicals in the supporting matrix and various radical forms produced by the interaction of non-completely reduced ions of gold (single charged) with the matrix atoms [23]. Figure 4 shows first three features originating from the stoichiometric reduction process with further centrifugal extraction. The line with $g=2.062$ has a fine structure as a sequence of individual peaks which is presented at the zooming of the central part of the plots (Fig. 4, insets). The synthesis with aluminum oxyhydrate and tannin was reported to produce Au-containing clusters with the size of several nanometers [28], which is of the same order of magnitude as cyclotron radius for the magnetic field of X-band EPR $(\sim 0.3 \mathrm{mT})$. Hence these peculiarities can be interpreted as a response from the excitation of subsequent electron cyclotron states. The evolution of the EPR spectra versus temperature points to the mutual interrelation of the signals observed in these samples, Fig. 4(b)-(c). The spectra are registered under various temperatures: $300 \mathrm{~K}$ (solid lines) and 200 
$\mathrm{K}$ (dashed lines). Insets: zooming of the central parts of the spectra. Bottom of each figure: constituents of the spectra-superpositions (Lorentz differential forms). Particularly, the amplitude and width of the peaks change with temperature: intensity of some lines increases and that of other decreases. The samples prepared by various routes have different set of lines and their behavior also differs.

The sample extracted from the solution via salting-out in dioxane

There are two signals with values of $g$-factors of 2.17 and 2.062. The line corresponding to the two-valent state has higher intensity than that of the line associated with zero-valent gold clusters with a fine structure revealed at more detail consideration (Fig. 4(b), inset). The intensities of these lines are mutually redistributed at low temperature: the line of the two-valent gold becomes weaker and wider whereas the zero valent line intensity increases.

The sample extracted from the solution with aluminum excess

The EPR of this sample, Fig. 4(c), contains lines of two-valent gold, clusters of the reduced gold and line with $g<g_{\mathrm{e}}$. The latter originates from complex formation with radicals and single-valent gold. The presence of the aluminate component is found to facilitate the yield of the Au-cluster, which is reflected by the integral intensity of the line with $2.062 g$-factor. This line dominates at low temperature. Thus all lines are mutually connected and originated from the nanospecies localized in the same supporting structure. The vibrational modes of the supporting environment hamper the exchange interaction between these nanospecies (Fig. 2, inset). Their magnetic moments interact only at low temperatures producing a spin coherency between them with common yield to the integral signal characteristic for Au-nanocluster.

The sample extracted from the solution with lack of the reducing agent

The EPR spectrum, Fig. 4(d), contains three components with $g=2.17, g=2.062, g=2.0048$ at room temperature, whereas at low temperature (200 K) the line with $g=2.17$ disappears which is explained as discussed in previous paragraph with the only difference that the intensity of the zero-valent gold line does not increase with reducing the temperature. The latter allows one to conclude that the clusters of the reduced gold lose their paramagnetic properties at low temperatures due to joining the particles into community with new electronic structure when the conditions of odd number of atoms cannot be already met.

Such a big difference in the behavior of two systems with aluminum excess and the lack of the reducing agent, Fig. 4(c)-(d) respectively, points to the difference in the structure of the underlying nanospecies. Eventually, the presence of the oxo-hydroxo aluminate form produces the conditions for the additional selection of the nanospecies, which is confirmed by the properties of these forms as Brownian motors. The action of these nanomechanisms facilitates the formation of the powerful system of clusters containing completely reduced gold atoms.

\section{Conclusion}

The paramagnetic nanospecies fabricated in this study demonstrate similar character for the formation of nanoclusters with a labile macromolecule as a supporting matrix. Both systems (silver nanospecies in hybride matrix of polyacrylic chains and gold-containing clusters within the labile matrix of tannin-citrate-oxohydrate of aluminum) reveal common features in the algorithm of the technological process for the formation of nanospecies: initially metal ions are captured by the supporting matrix, then the reducing agent acts producing an hierarchical system containing the metal atoms in the matrix. The lability of the supporting molecular system facilitates the interatomic interactions. The analysis of the evolution of the forms revealed for both systems in the EPR spectra yields the following conclusions:

- the EPR spectra have several peculiarities at room temperature. The EPR spectra for the samples with silver in polyacrylic matrix have two types of signals: anisotropic with $g_{\|} \sim 2.036$ and $g_{\perp} \sim 2.02$; and isotropic with $g \sim 2.03, \Delta B \sim 7 \mathrm{mT}$. The system of gold in the matrix of tannin-citrate- aluminum oxohydrate produces EPR signals with lines of four types with $g=2.17, g=2.062, g=2.0048$ and $g<2$, with their integral intensities being dependent on the preparation conditions. In addition, the line with $g=2.062$ (attributed to the cluster with odd number of gold reduced atoms) has a fine structure as a sequence of individual weak peaks which are, presumably, the result of cyclotron resonance.

- the adjacent paramagnetic nanospecies participate in exchange interaction at low temperatures, which is suppressed at higher temperatures by the vibrational modes in the molecule-matrix. This behavior is revealed as changes in the amplitude of certain lines of the EPR spectrum. Some lines disappear while reducing temperature.

- the form of the nanospecies is determined by a supporting matrix. In particular, the polymer chains in the system of silver in polyacrylic matrix guide the orientation growth of the silver nanospecies responding as anisotropic EPR signal. This signal modifies at lower temperatures: one form with $g_{\|}<g_{\perp}$ transforms into the other with $g_{\|}>g_{\perp}$. This transition takes place at $\sim 220 \mathrm{~K}$, and is, likely, attributed to phase transition of water embedded in nanopores and between the polymer chains.

\section{REFERENCES}

[1] J. Kudr, Y. Haddad, L. Richtera, Z. Heger, M. Cernak, V. Adam, O. Zitka, "Magnetic Nanoparticles: From Design and Synthesis to Real World Applications", Nanomaterials, vol. 7, no. 9, pp. 243-272, 2017. 
[2] Q. A. Pankhurst, J. Connolly, S. K. Jones, J. Dobson, "Application of magnetic nanoparticles in biomedicine", J. Phys. D Appl. Phys., vol. 36, pp. 167-181, 2003.

[3] J. Gomez-Pastora, S. Dominguez, E. Bringas, M. J. Rivero, I. Ortiz, D. D. Dionysiou, "Review and perspectives on the use of magnetic nanophotocatalysts (MNPCs) in water treatment", Chemical Engineering Journal, vol. 310(2), pp. 406-427, 2017.

[4] E. J. Werner, A. Datta, C. J. Jocher, K. N. Raymond, "High-Relaxivity MRI Contrast Agents: Where Coordination Chemistry Meets Medical Imaging", Angew. Chemie. Intl. Ed., vol. 47, pp. 8568-8580, 2008.

[5] A. Kovalenko, R. Singh Yadav, J. Pospisil, O. Zmeskal, D. Karashanova, P. Heinrichova, M. Vala, J. Havlica, M. Weiter, "Towards improved efficiency of bulk-heterojunction solar cells using various spinel ferrite magnetic nanoparticles", Organic Electronics, vol. 39, pp. 118-126, 2016.

[6] V. V. Kislyuk, O. Ya. Kuznetsova, "Flexible organic photovoltaics", High Technologies, vol. 4(40), pp. 492-495, 2018.

[7] R. Becker, W. Döring, "Kinetische Behandlung der Keimbildung in übersättigten Dämpfen", Annalen der Physik, vol. 416(8), pp. 719-752, 1935.

[8] V. K. LaMer, R. H. Dinegar, "Theory, Production and Mechanism of Formation of Monodispersed Hydrosols", J. Am. Chem. Soc., vol. 72(11), pp. 4847-4854, 1950.

[9] B. Derjaguin, L. D. Landau, "Theory of the Stability of Strongly Charged Lyophobic Sols and of the Adhesion of Strongly Charged Particles in Solutions of Electrolytes", Acta Physicochimica U.R.S.S., vol. 14, pp. 633-662, 1941.

[10] E. J. W. Verwey, J. Th. G. Overbeek, "Theory of the Stability of Lyophobic Colloids", Elsevier, Amsterdam, 1948.

[11] Yu. Mikhlin, A. Karacharov, M. Likhatski, T. Podlipskaya, I. Zizakc, "Direct observation of liquid pre-crystallization intermediates during the reduction of aqueous tetrachloroaurate by sulfide ions", Phys. Chem. Chem. Phys., vol. 16, pp. 4538-4543, 2014.

[12] R. J. White, R. Luque, V. L. Budarin, J. H. Clarka, D. J. Macquarriea, "Supported metal nanoparticles on porous materials. Methods and applications", Chem. Soc. Rev., vol. 38(2), pp. 481-494, 2009.

[13] Z. Qu, E. Roduner, "In situ ESR study of gold supported on NaY zeolite", Asia-Pac. J. Chem. Eng., vol. 4, pp. 602-606, 2009.

[14] Ju. Zhang, J. E. Kielbasa, D. L. Carroll, "Nanostructure of the nanopores in anodic aluminum oxide films used as template to fabricate Ag nanowires", J. Mater. Res., vol. 24(5), pp. 1735-1740, 2009.

[15] R. Feynman, R. Leighton, M. Sands, "The Feynman Lectures on Physics", Addison-Wesley: Reading, MA, 1963.

[16] G. P. Suarez, M. Hoyuelos, D. R. Chialvo, "Invited review: Fluctuation-induced transport from the very small to the very large scales", Papers in Physics, vol. 8, pp. 080004-1-080004-11, 2016.

[17] Yu. I. Sukharev, I. J. Apalikova, V. O. Apalikov, J. D. Meshcheryakova, O. V. Leonova, I. A. Zasoba, "Periodic formation current self-organization in oxyhydrate environment", Tomsk State University Journal of Chemistry, vol. 1(3), pp. 45-59, 2016.

[18] V. K. Vanag, "Study of spatially extended dynamical systems using probabilistic cellular automata", Phys. Usp., vol. 42(5), pp. 413-434, 1999 .

[19] A. Abedini, A. R. Daud, M. A. A. Hamid, N. K. Othman, E. Saion, "A review on radiation-induced nucleation and growth of colloidal metallic nanoparticles", Nanoscale Res Lett., vol. 8(1), pp. 474-484, 2013.

[20] Ya. Shang, Ch. Min, Ju. Hu, T. Wang, H. Liu, Yi. Hu, "Synthesis of gold nanoparticles by reduction of $\mathrm{HAuCl}_{4}$ under UV irradiation", Solid State Sciences, vol. 15, pp. 17-23, 2013.

[21] H. K. Cho, Iu. Bondar, Do H. Han, Y. K. Kwon, "Proton exchange membranes prepared by radiation-induced graft copolymerization from binary monomer mixtures onto poly (tetrafluoroethylene-co-hexafluoropropylene) film", Nuclear Instruments and Methods in Physics Research B, vol. 268, pp. 1588-1593, 2010.

[22] M. Gilaki, "Synthetic of Magnetic $\mathrm{Al} / \mathrm{Au}$ Nanoparticles by Co-Reduction of $\mathrm{Au}^{3+}$ and $\mathrm{Al}^{3+}$ Metal Salts", Pakistan Journal of Biological Sciences, vol. 13(16), pp. 809-813, 2010.

[23] V. V. Kislyuk, A. K. Melnyk, N. I. Buryak, V. V. Trachevskij, "NMR study of Au/Al nanosyatems in solution", J. Electr. Eng., 2019, (current issue).

24] N. Perkas, G. Amirian, G. Applerot, E. Efendiev, Y. Kaganovskii, A. V. Ghule, B. J. Chen, Y. C. Ling, A. Gedanken, "Depositing silver nanoparticles on/in a glass slide by the sonochemical method", Nanotechnology, vol. 19(43), pp. 435604-1-435604 $-9,2008$.

25] A. Baldansuren, E. Roduner, "EPR experiments of Ag species supported on NaA", Chem. Phys. Lett., vol. 473, pp. 135-137, 2009.

[26] E. B. Moore, V. Molinero, "Structural transformation in supercooled water controls the crystallization rate of ice", Nature, vol. 479, pp. 506-508, 2011.

[27] E. Mamontov, Y. Yue, J. Bahadur, J. Guo, C. I. Contescu, N. C. Gallego, Y. B. Melnichenko, "Hydration level dependence of the microscopic dynamics of water adsorbed in ultramicroporous carbon", Carbon, vol. 111, pp. 705-712, 2017.

28] V. Kislyuk, A. K. Melnyk, N. I. Buryak, V. V. Trachevskij, "Quantum confinement features in EPR and UV-vis spectra of $\mathrm{Al} / \mathrm{Au}$ nanosystems", Journal of Nano- and Electronic Physics, vol. 11(4), pp. 04037-1-04037-4, 2019.

Received 19 March 2019

Victor Kislyuk is a Senior Researcher of G.V. Kurdyumov Institute of Metal Physics, National Academy of Sciences of Ukraine. PhD degree was awarded in 1999, the grade Associate Professor since 2006. Research interests and expertise: organic conducting materials and composites for electronics, in particular for photovoltaics (PV), characterization of materials and structures by spectroscopic techniques, UV-vis absorption, fluorescence, NMR, EPR, PV performance

Andrii Melnyk received his Master degree from the National Technical University of Ukraine "Kiev Politechnical Institute" in 1996. He is Research Assistant at the Institute for Sorption and Endoecology Problems of the NAS of Ukraine since 2009. His professional interests include carbonbased nanomaterials, nanocomposites, spin probes and spinlabeled molecules, magnetic films and nanoparticles, transition metal ions, defects and impurities in crystalline and amorphous solids.

Yuliia Bondar is a senior scientist of Institute of Environmental Geochemistry of National Academy of Sciences of Ukraine. She graduated from Department of Physical Chemistry of the National Taras Shevchenko University of Kyiv (Ukraine) and began to work in the Institute of Geochemistry, Mineralogy and Ore Formation of NAS of Ukraine (Kyiv, Ukraine). In 1994 she got PhD degree in Geochemistry and latter Senior Scientist degree. Current interests: radiationchemical modification of polymers; synthesis of nanocomposite polymer materials; synthesis and application of nanocomposites as adsorbents for sensing and selective removal of heavy metals and radionuclides from contaminated waters.

Volodymyr Trachevskij PhD since 1975. Head of laboratory of Technological center of National Academy of Sciences of Ukraine. Expertise in diagnostics of self organization in condensed matter by spectroscopic techniques including NMR, EPR, IR, UV-vis, XPS. The author of more than 300 articles and 15 books. 